ARTICLE

Received 26 May 2014 | Accepted 24 Jul 2014 | Published 10 Sep 2014

DOI: $10.1038 /$ ncomms5801

OPEN

\title{
Crystal structure of a common GPCR-binding interface for $G$ protein and arrestin
}

Michal Szczepek ${ }^{1}$, Florent Beyrière ${ }^{1}$, Klaus Peter Hofmann ${ }^{1,2}$, Matthias Elgeti ${ }^{1}{ }^{\dagger}$, Roman Kazmin ${ }^{1}$, Alexander Rose ${ }^{1,3}$, Franz J. Bartl1,2, David von Stetten ${ }^{4}$, Martin Heck ${ }^{1}$, Martha E. Sommer ${ }^{1}$, Peter W. Hildebrand ${ }^{1,3} \&$ Patrick Scheerer ${ }^{1,5}$

G-protein-coupled receptors (GPCRs) transmit extracellular signals to activate intracellular heterotrimeric $G$ proteins $(G \alpha \beta \gamma)$ and arrestins. For $G$ protein signalling, the $G \alpha C$-terminus $(\mathrm{G} \alpha \mathrm{CT})$ binds to a cytoplasmic crevice of the receptor that opens upon activation. A consensus motif is shared among $\mathrm{G} \alpha \mathrm{CT}$ from the $\mathrm{G}_{i} / \mathrm{G}_{t}$ family and the 'finger loop' region (ArrFL1-4) of all four arrestins. Here we present a $2.75 \AA$ crystal structure of ArrFL-1, a peptide analogue of the finger loop of rod photoreceptor arrestin, in complex with the prototypical GPCR rhodopsin. Functional binding of ArrFL to the receptor was confirmed by ultraviolet-visible absorption spectroscopy, competitive binding assays and Fourier transform infrared spectroscopy. For both $\mathrm{G} \alpha \mathrm{CT}$ and ArrFL, binding to the receptor crevice induces a similar reverse turn structure, although significant structural differences are seen at the rim of the binding crevice. Our results reflect both the common receptor-binding interface and the divergent biological functions of $\mathrm{G}$ proteins and arrestins.

\footnotetext{
${ }^{1}$ Institut für Medizinische Physik und Biophysik (CC2), Charité-Universitätsmedizin Berlin, Charitéplatz 1, D-10117 Berlin, Germany. ${ }^{2}$ Zentrum für Biophysik und Bioinformatik, Humboldt-Universität zu Berlin, Invalidenstrasse 42, D-10115 Berlin, Germany. ${ }^{3}$ Institut für Medizinische Physik und Biophysik (CC2), AG Proteilnformatics, Charité-Universitätsmedizin Berlin, Charitéplatz 1, D-10117 Berlin, Germany. ${ }^{4}$ Structural Biology Group, European Synchrotron Radiation Facility, CS 40220, F-38043 Grenoble, France. ${ }^{5}$ Institut für Medizinische Physik und Biophysik (CC2), AG Protein X-ray Crystallography, Charité-Universitätsmedizin Berlin, Charitéplatz 1, D-10117 Berlin, Germany. †Present address: Department of Chemistry and Biochemistry, Jules Stein Eye Institute, University of California, Los Angeles, California 90095-700, USA. Correspondence and requests for materials should be addressed to P.S. (email: patrick.scheerer@charite.de).
} 
$\mathrm{T}$ he G-protein-coupled receptor (GPCR) rhodopsin is composed of a seven transmembrane helix (7-TM) bundle and a covalently attached retinal ligand, which undergoes light-induced isomerization to yield the photoproduct metarhodopsin II and the $\mathrm{R}^{\star}$ conformation capable of coupling to the $\mathrm{G}$ protein transducin $\left(\mathrm{G}_{t}\right)^{1}$ (Fig. 1). The $\mathrm{G}_{t} \alpha$ C-terminus $\left(\mathrm{G}_{t} \alpha \mathrm{CT}\right)$ is disordered in uncomplexed $G_{t}$ but forms an $\alpha$-helix terminated by a reverse turn (C-cap) upon interaction with the $\mathrm{R}^{*}$ cytoplasmic crevice ${ }^{2-6}$. Signal transduction is followed by signal shut-off: rhodopsin kinase phosphorylates the $\mathrm{R}^{*} \mathrm{C}$-terminus, which recruits rod photoreceptor arrestin for high-affinity interaction with $\mathrm{R}^{\star}$ (ref. 7). Interaction of arrestin with the phosphorylated $\mathrm{R}^{\star} \mathrm{C}$-terminus displaces the long $\mathrm{C}$-terminal tail (C-tail) of arrestin. As a result, the arrestin basal state, which is structurally similar in all four arrestins ${ }^{8-11}$, is activated for $\mathrm{R}^{*}$ binding (Fig. 1). Insight into the structure of activated arrestin was recently gained by crystal structures of pre-activated C-terminally truncated rod photoreceptor arrestin $(\mathrm{p} 44)^{12}$ or $\beta$-arrestin-1 co-crystallized with a synthetic phosphorylated peptide derived from V2 vasopressin receptor C-terminus ${ }^{13}$. In comparison to basal arrestin, pre-activated arrestin shows rearrangements of binding loops in the central crest region, including the so-called finger loop (ArrFL) $)^{14-19}$, suggesting that C-tail displacement releases these loops for interaction with $\mathrm{R}^{*}$ (Fig. 1). The present work was motivated by the observation of a consensus sequence motif, (E/D)x(I/L)xxxGL, which is conserved in $\mathrm{G}_{\mathrm{t}} \alpha \mathrm{CT}$ and the finger loop of all four arrestins (Fig. 1, inset and Supplementary Fig. 1). We address the question of how arrestins employ the cytoplasmic crevice within the 7-TM bundle of the active receptor $\mathrm{R}^{\star}$ for the high-affinity interaction necessary to block $G$ protein binding and/or stimulate arrestin-mediated signalling ${ }^{13}$. Co-crystals of active rhodopsin $\left(R^{\star}\right)$ with a peptide analogue derived from the finger loop of the rod photoreceptor arrestin (ArrFL-1) were obtained, which diffracted up to $2.75 \AA$ resolution. Similar to $\mathrm{G}_{t} \alpha \mathrm{CT}^{3}$, ArrFL binds to the cytoplasmic crevice of $\mathrm{R}^{*}$ with a $\mathrm{C}$-terminal reverse turn-like structure that involves the conserved $\mathrm{E}(\mathrm{D}) \mathrm{RY}$ motif of $\mathrm{R}^{*}$. Consistently, functional experiments demonstrate similar $R^{*}$-binding properties of $G_{i} / G_{t}$ and ArrFL peptides. However, in ArrFL-1, the interaction with TM5/6 is partially replaced by interactions with TM7/H8 (specifically the $\operatorname{NPxxY}(\mathrm{x})_{5,6} \mathrm{~F}$ motif). We discuss if and how the similarities between $\mathrm{G} \alpha \mathrm{CT}$ and ArrFL reflect common mechanisms of signal transfer between receptor and interacting proteins. At the same time, the differences between $\mathrm{G} \alpha \mathrm{CT}$ and ArrFL might indicate the different biological functions of $G$ protein and arrestin.

\section{Results}

The structure of Ops ${ }^{\star}$-ArrFL-1. We co-crystallized $\mathrm{R}^{\star}$ (lightactivated rhodopsin (Meta II) or the active form of the apoprotein, $\mathrm{Ops}^{\star}$ ) with ArrFL peptides derived from rod photoreceptor arrestin $\left({ }^{67}\right.$ YGQEDIDVMGL ${ }^{77}=$ ArrFL- 1$), \beta$-arrestin- 1 and $\beta$ arrestin-2 ( ${ }^{63 / 64}$ YGREDLDVLGL ${ }^{73 / 74}=$ ArrFL-2/3). Our first strategy was to illuminate a mixture of rhodopsin and ArrFL peptides directly before crystallization (Supplementary Figs 2 and 3). The resulting crystal structures (Meta II-ArrFL) showed weak electron density for both the all-trans-retinal ligand and the peptides (only three residues from the C-terminus were resolved). This problem was most likely due to structural heterogeneity within the crystal, which we overcame by co-crystallizating Ops ${ }^{\star}$

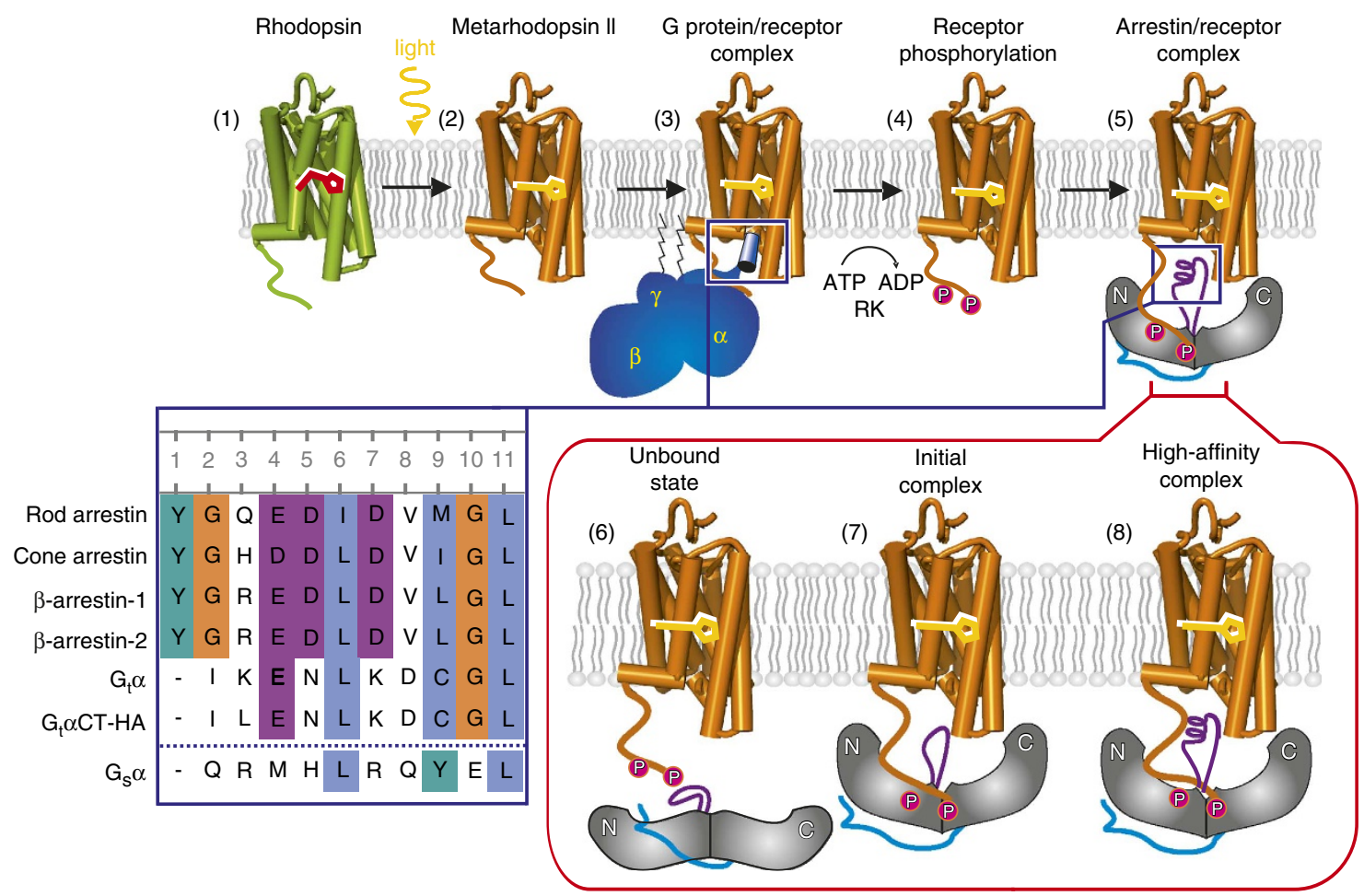

Figure 1 | Overview of rhodopsin signal transduction and deactivation. (1) Dark-state bovine rhodopsin with inverse agonist 11-cis-retinal. (2) Light-activation and formation of metarhodopsin II (Meta II) with agonist all-trans-retinal. (3) Coupling to the heterotrimeric G protein. (4) Phosphorylation of the activated receptor by its specific kinase. (5) Rod photoreceptor arrestin binding to the phosphorylated receptor. Inset (red box): (6) Basal arrestin approaches phosphorylated Meta II. (7) Interaction with receptor-attached phosphates activates arrestin. (8) Tight binding of the receptor by arrestin (induces further structural changes in arrestin finger loop). Inset (indigo box): Sequence alignment of proposed common sequence motif: rod photoreceptor arrestin (Arr1, residues 68-77), cone photoreceptor arrestin (Arr4, residues 63-72), $\beta$-arrestin- 1 (Arr2, residues 64-73), $\beta$-arrestin-2 (Arr3, residues 65-74), wild-type $\mathrm{G}_{t} \alpha C T$ (residues 340-349), high-affinity variant $\mathrm{G}_{t} \alpha C T-H A$ (residues 340-349), $\mathrm{G}_{\mathrm{s}} \alpha$ (residues 385-394). 
and ArrFL peptides. Best results were obtained with ArrFL-1, which diffracted up to $2.75 \AA$ resolution (Fig. 2, Table 1, Supplementary Figs 2-4). The Ops ${ }^{\star}-$ ArrFL-1 structure contains amino acids $2-326$ of opsin, including the 7-TM helices connected by extracellular (EL1-EL3) and cytoplasmic (CL1-CL3) loops, and the cytoplasmic helix 8 (H8), which runs along the membrane surface (Fig. 2a, Supplementary Fig. 4). The last 22 C-terminal amino acids of $\mathrm{Ops}^{\star}$ were not resolved, presumably due to their high flexibility. The atomic structure of the ArrFL-1 peptide (residues 71-77) was solved from a continuous electron density located within the cytoplasmic crevice of $\mathrm{R}^{*}$ (Supplementary Fig. 4), whereas the N-terminal extended part of the peptide (residues 71-73) showed weaker electron density (Supplementary Table 1). Well-defined electron density for a detergent molecule, $\beta$-D-octylglucopyranoside, is present within the ligand-binding pocket of $\mathrm{R}^{\star}$ (Supplementary Fig. 5$)^{20}$. Note that the overall structure of the receptor in all Meta II-ArrFL peptide complexes are essentially the same as the Ops ${ }^{*}-$ ArrFL-1 structure.

The $\mathrm{R}^{*}$ structure presented here is similar to previous structures of active rhodopsin (Ops ${ }^{\star}$ or Meta II), alone $e^{4,21}$ or bound to high-affinity variants of the $\mathrm{G}_{\mathrm{t}} \alpha \mathrm{CT}$ peptide $\mathrm{e}^{3,4,22}$ (Supplementary Fig. 6). The Ops ${ }^{\star}-\mathrm{ArrFL}_{-1}$ complex displays the hallmarks of GPCR activation, including the outward tilt of TM6 $(>7 \AA)$ and inward motion of TM5, that open the cytoplasmic receptor surface and form a crevice for ArrFL-1 binding. The C-terminal residues 73-77 of ArrFL-1, which were also predicted by mutagenesis as a key binding site with $\mathrm{R}^{*}$ (ref. 17, fold similar to the corresponding section of $\mathrm{G}_{\mathrm{t}} \alpha \mathrm{CT}$ (Supplementary Fig. 6). The conformation of ArrFL-1 is reminiscent of the helical structure predicted by other studies $^{16,23}$. The presence of Gly76 facilitates the formation of a reverse turn-like structure, similar to the typical $\alpha$ L-type C-capping motif (C-cap) previously observed for $\mathrm{G}_{t} \alpha \mathrm{CT}^{3}$ (Supplementary Fig. 6). In ArrFL-1, formation of the reverse turn-like structure is made possible by the strong internal hydrogen bonding from Asp73 (main chain carbonyl, MC-O) to Leu77 (main chain amide, MC-N; Fig. 3c) and the flexibility of Gly76, facilitating a left-handed turn conformation. Despite this similarity, the positioning of ArrFL and $\mathrm{G}_{t} \alpha \mathrm{CT}$ differ because of the fact that ArrFL is part of a loop connecting two $\beta$-strands within arrestin and not the end of a helix like $\mathrm{G}_{\mathrm{t}} \alpha \mathrm{CT}$. The N-terminal part of ArrFL-1 (residues 71-72) is non-helical and extends in the direction of CL2 of Ops* (Fig. 2). Note that residues $67-70$ are not resolved. The structure also indicates that the C-terminal extension of the finger loop would be located between TM6 and TM7/H8. This arrangement would place the cytoplasmic ends of TM1, TM2 and TM7/H8 roughly within the $\mathrm{N}$-domain vestibule of arrestin, whereas the ends of TM3, TM5 and TM6 would be positioned near the central crest of arrestin.

ArrFL-1 forms specific contacts with two highly conserved motifs in the receptor: Arg $135^{3.50}$ within E(D)RY and Lys $311^{8.48}$

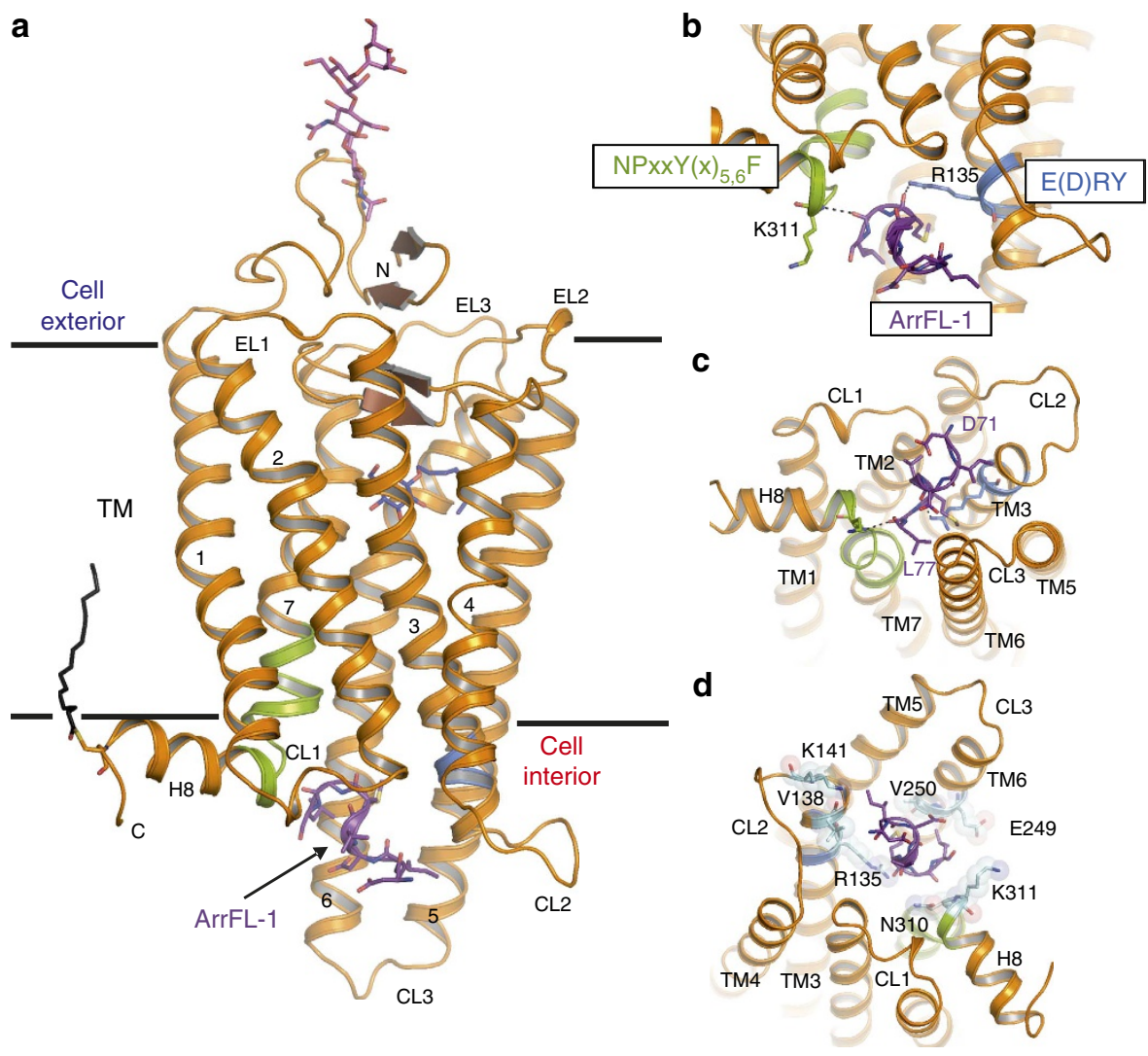

Figure 2 | Overall structure of the Ops ${ }^{\star}$-ArrFL-1 complex. (a) The active receptor Ops ${ }^{\star}$ (orange) and the ArrFL-1 peptide (purple) are shown in ribbon representation, and the ArrFL-1 side chains are shown as sticks. Oligosaccharides at Asn15 ${ }^{\mathrm{NT}}$ (pink) and a palmitoyl chain at Cys3238.60 (black) are presented as sticks. The detergent molecule, $\beta$-D-octylglucopyranoside (blue), is shown within the ligand-binding pocket as sticks. (b) Side view and (c) top view of the binding crevice of Ops ${ }^{\star}$. Major hydrogen bonding interactions of Ops ${ }^{\star}$ with ArrFL-1 are shown between Leu77 and Lys311 8.48 from the $\operatorname{NPxxY}(\mathrm{x})_{5,6} \mathrm{~F}$ motif (green), and Met75 and Arg1353.50 from the E(D)RY motif (blue). (d) Additional rotated top view of the binding crevice of Ops ${ }^{\star}$ Hydrophobic interactions between ArrFL-1 and residues in the binding interface of Ops ${ }^{\star}$ protein moiety are shown as sticks and spheres (light-blue). Arg135 3.50 of TM3, Val138 3.53 , Lys $141^{\mathrm{CL} 2}$ of CL2, Glu249 6.32 , Val2506.33 of TM6 and Asn310 8.47 , Lys311 8.48 of TM7/H8 are in van der Waals contact to ArrFL-1 (that is, separated by less than $4 \AA$ ). 


\section{Table 1 | Data collection and refinement statistics.}

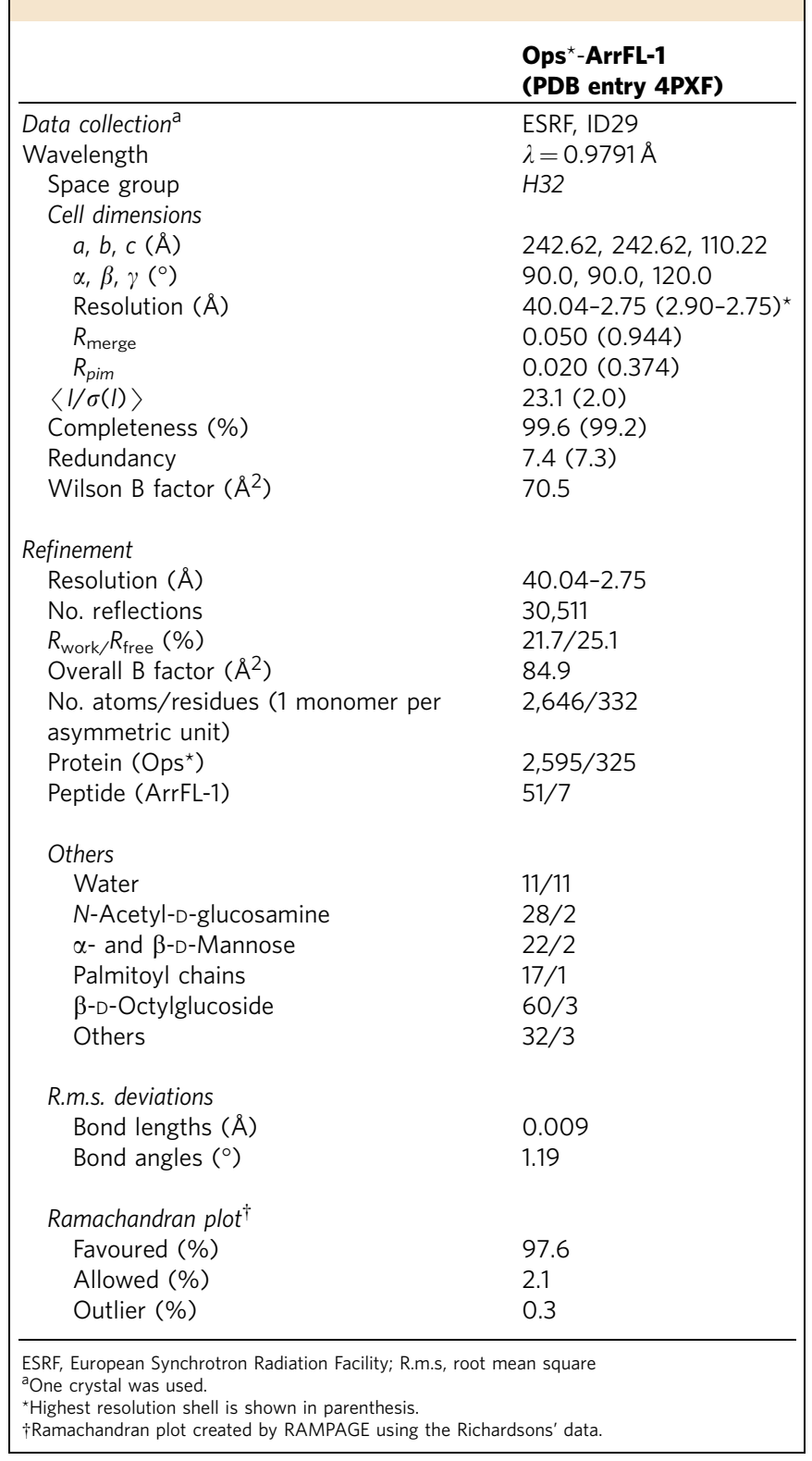

within $\operatorname{NPxxY}(\mathrm{x})_{5,6} \mathrm{~F}$ (Fig. 2; note: superscript following residue number refers to Ballesteros-Weinstein numbering, see Full Methods). A hydrogen bond (distance $2.85 \AA$ ) is formed between Met75 (MC-O) of ArrFL-1 and the guanidine group of Arg135.50 in $\mathrm{R}^{*}$ (Figs $2 \mathrm{~b}$ and $3 \mathrm{c}$, Supplementary Figs 7,8 and 11 ). This bond is similar to that observed for Cys347 in $\mathrm{G}_{\mathrm{t}} \alpha \mathrm{CT}$ and $\operatorname{Arg} 135^{3.50}$ (Fig. 3b). Lys $311^{8.48}$ within the $\operatorname{NPxxY}(\mathrm{x})_{5,6} \mathrm{~F}$ motif forms a distinct hydrogen bond with ArrFL-1: Lys311 8.48 (MC-N) to Gly76 (MC-O) (distance $2.97 \AA$ A Figs $2 \mathrm{~b}$ and 3c, Supplementary Figs 7,8 and 11 ). In contrast, Gln $312^{8.49}$ (side chain) makes a hydrogen bond with Lys345 (MC-O) of $\mathrm{G}_{\mathrm{t}} \alpha \mathrm{CT}$ (Fig. 3b, Supplementary Figs. 7 and 8). Both ArrFL-1 and $\mathrm{G}_{\mathrm{t}} \alpha \mathrm{CT}$ are in van der Waals contact with Asn $310^{8.47}$ (Fig. 2d, Supplementary Figs 7 and 8 ). Note that no strong interactions of polar side chains of ArrFL-1 with the receptor are visible. Furthermore, additional van der Waals contacts are formed between Ile72 on ArrFL-1 and Val138 $8^{3.53}$ and Lys $141^{\mathrm{CL} 2}$. In comparison, $\mathrm{G}_{\mathrm{t}} \alpha \mathrm{CT}$ makes contacts with Val138 $8^{3.53}$, Val139 $9^{3.54}$ and Lys141 ${ }^{\mathrm{CL} 2}$. Overall, the contacts observed between ArrFL-1 and the receptor loops are consistent with previous studies implicating receptor loops CL1 and CL2 in arrestin binding ${ }^{19,24-26}$. With respect to receptor binding, the main difference between ArrFL- 1 and $\mathrm{G}_{\mathrm{t}} \alpha \mathrm{CT}$ is the contribution of TM5/6. ArrFL-1 makes only minor contacts to TM6 (Leu77 to Glu249 $9^{6.32}$, Val250 $0^{6.33}$ ) and no contacts to TM5. In contrast, extensive hydrophobic interactions exist between several amino acid side chains of $\mathrm{G}_{t} \alpha \mathrm{CT}$ (Ile340, Leu341, Phe350) and the 'hydrophobic wall' formed by TM5 (Leu226 $6^{5.61}$, Val230 CL3, Ala233 CL3) and TM6 (Thr242 CL3, Thr243 ${ }^{\text {CL3 }, ~ A l a 246 ~}{ }^{6.29}$, Val2506.33; Supplementary Fig. 7). In essence, the interactions of ArrFL-1 with TM5/6 are reduced as compared with $\mathrm{G}_{t} \alpha \mathrm{CT}$, but those with TM7/H8 $\left(\mathrm{NPxxY}(\mathrm{x})_{5,6} \mathrm{~F}\right.$ motif) and TM3 (E(D)RY motif) are preserved (Fig. $3 c$ and Supplementary Fig. 8). This finding is consistent with recent studies localizing G-protein- and arrestin-specific binding determinants to TM6 and TM7/H8, respectively ${ }^{27-29}$.

Functional characterization of peptides. To verify the functional relevance of the observed peptide binding to the receptor crevice (Fig. 4a, b), we applied three different well-established functional assays. These assays were performed in native disc membranes and not the detergent system used for crystallization, as the native membrane offers many advantages to observe the interactions of peptides with the different functional forms of rhodopsin. In the first assay, we light-activated rhodopsin under conditions favouring Meta I, the inactive precursor of the active species Meta II. Meta I and Meta II can be differentiated by ultraviolet-visible absorption spectroscopy due to their different absorbance maxima. Like both full-length rod photoreceptor arrestin (ref. 30) and $\mathrm{G}_{\mathrm{t}} \alpha \mathrm{CT}^{31}$, ArrFL-1 stabilized the 380-nm-absorbing Meta II species over Meta I, showing that ArrFL-1 functionally binds the active state of the receptor. Titration analysis indicated $K_{\mathrm{D}}$ values of $2.4 \mu \mathrm{M}$ and $0.8 \mathrm{mM}$ for $\mathrm{G}_{\mathrm{t}} \alpha \mathrm{CT}$-HA (high-affinity version) and ArrFL-1, respectively (Fig. 4c, d). For comparison, the $K_{\mathrm{D}}$ values of the wild-type $\mathrm{G}_{\mathrm{t}} \alpha \mathrm{CT}$ and ArrFL-2/3 are 0.18 and $1.3 \mathrm{mM}$, respectively (Supplementary Fig. 9). In the second assay, we measured the ability of $\mathrm{G}_{\mathrm{t}} \alpha \mathrm{CT}$ and ArrFL peptides to compete against full-length rod photoreceptor arrestin for binding to phosphorylated opsin (OpsP) in membranes (Fig. 4e,f, Supplementary Fig. 9). The competition data yield $K_{\mathrm{D}}$ values of $320 \mu \mathrm{M}, 880 \mu \mathrm{M}, 1.7 \mathrm{mM}$ and $2.4 \mathrm{mM}$ for peptides $\mathrm{G}_{\mathrm{t}} \alpha \mathrm{CT}-\mathrm{HA}$, wild-type $\mathrm{G}_{\mathrm{t}} \alpha \mathrm{CT}$, ArrFL-1 and ArrFL-2/3, respectively. Importantly, these data indicate that full-length rod photoreceptor arrestin and ArrFL have a common receptorbinding site. The peptide derived from $G_{s} \alpha C T$ from the $G_{s}$ family, which does not share the common sequence motif with ArrFL and $\mathrm{G}_{\mathrm{t}} \alpha \mathrm{CT}$, did not stabilize Meta II and showed no competition for arrestin binding to phosphorylated opsin (Supplementary Fig. 9). In the third assay, Fourier transform infrared (FTIR) spectroscopy was used to monitor the effects of Meta II-ArrFL-1 complex formation in native membranes resulting in characteristic bands, which mostly appear in the structurally sensitive amide I and amide II regions of the FTIR spectrum ${ }^{32,33}$ (Supplementary Fig. 10). The effect of ArrFL-1 and ArrFL-2/3 on the $\mathrm{R}^{*}$ characteristic difference spectrum is similar to $G_{t} \alpha C T$, although the data suggest a somewhat smaller influence on the peptide structure due to binding ${ }^{32}$. The $\mathrm{G}_{\mathrm{s}} \alpha \mathrm{CT}$ shows essentially no effect on the difference spectrum, in accordance with its lack of binding in the other assays. In further FTIR experiments, we investigated the R135L mutant and its capability of binding ArrFL-1 peptide (Supplementary Fig. 10). We observed that the characteristic arginine bands are missing upon binding, which suggest an involvement of Arg135 in ArrFL-1 binding under these conditions. Altogether, FTIR data confirm that peptides with the consensus sequence $(\mathrm{E} / \mathrm{D}) \mathrm{x}(\mathrm{I} / \mathrm{L}) \mathrm{xxxGL}$ specifically bind and stabilize the active conformation of the receptor in a very 

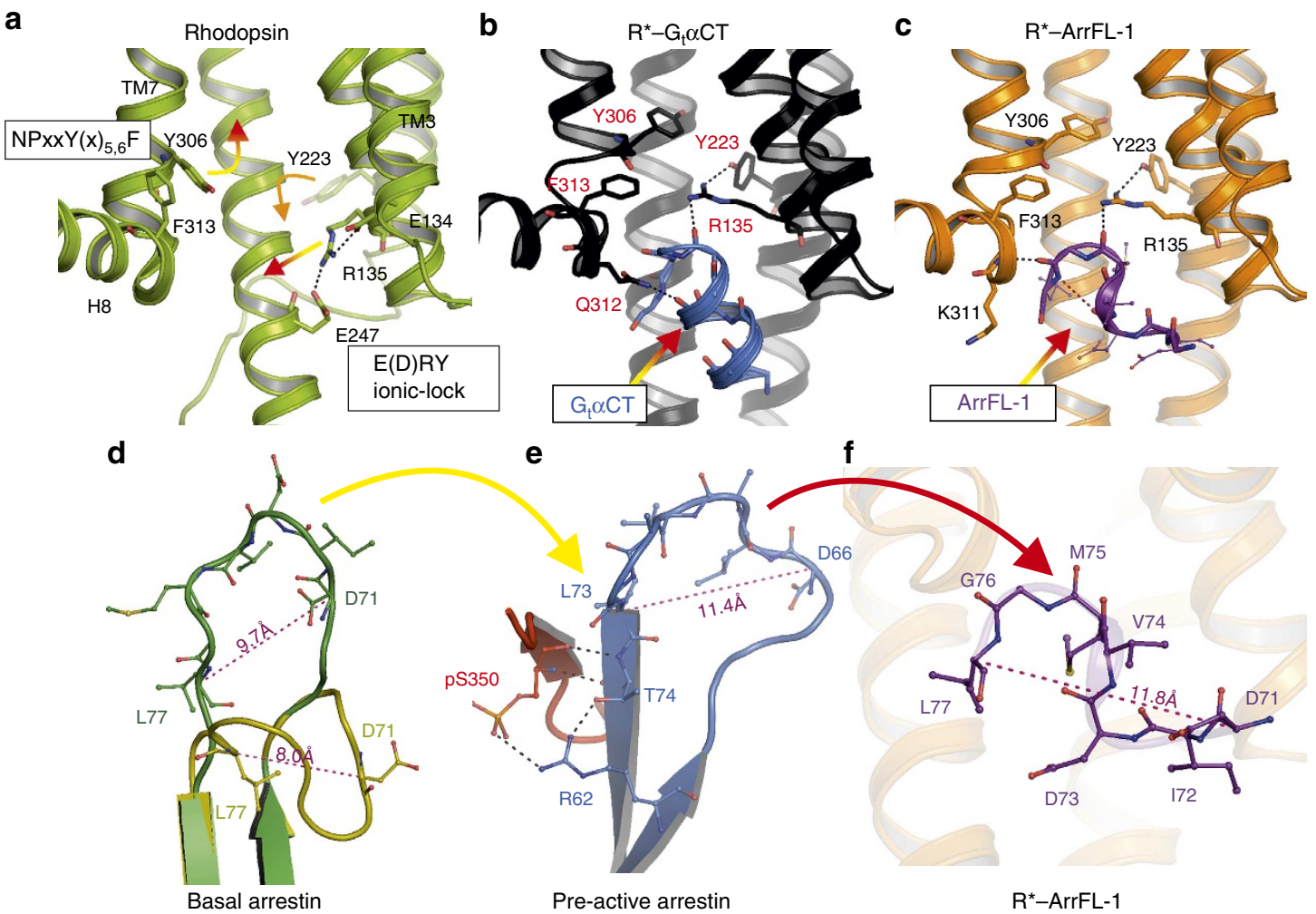

f

Pre-active arrestin

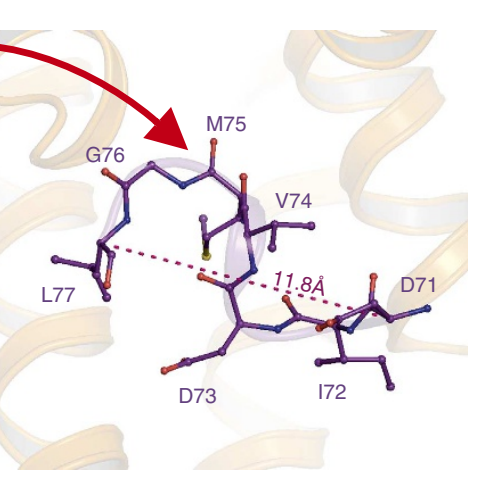

Figure 3 | The triple role of Arg135 $\mathbf{3 . 5 0}$ and changes in finger loop during arrestin activation and binding. (a) In dark-state rhodopsin (green), the helical bundle is bound together by multiple intra-helical interactions involving the highly conserved $E(D) R Y$ and $N P x x Y(x)_{5,6} F$ motifs. The arrows indicate side chain motions that occur upon receptor activation. (b) Binding of $\mathrm{G}_{t} \alpha \mathrm{CT}$ (blue) within the cytoplasmic crevice of $\mathrm{R}^{\star}$ (black). Main interactions include hydrogen bonding from Arg135.50 to the C-cap, and extensive hydrophobic interactions along the inner face of TM5/TM6. (c) Binding of ArrFL-1 (purple) within the cytoplasmic crevice of $\mathrm{R}^{\star}$ (orange). Main interactions include hydrogen bonding from Arg135 3.50 to the reverse turn like structure and Lys3118.48 to the GL sequence of ArrFL-1. (d) Finger loop in basal rod photoreceptor arrestin (PDB entry 1CF1) in 'closed' (olive) and 'open' (dark-green) conformations. (e) Finger loop in $\beta$-arrestin-1 (blue) bound to peptide analogue of the phosphorylated receptor C-terminus (red; PDB entry 4JQI). (f) Finger loop peptide ArrFL-1 (purple) bound within the crevice of $R^{\star}$. In $\mathbf{d - f}$, the backbone $C \alpha-C \alpha$ distance between Asp71/67 and Leu77/73 (numbering refers to rod photoreceptor arrestin/ $\beta$-arrestin- 1$)$ is indicated $(\AA)$.

similar manner as $\mathrm{G}_{\mathrm{t}} \alpha \mathrm{CT}$. It remains to be determined whether, like $\mathrm{G}_{\mathrm{t}} \alpha \mathrm{CT}$, ArrFL-1 discriminates between different conformational substates of Meta II (ref. 33).

\section{Discussion}

By the common $(\mathrm{E} / \mathrm{D}) \mathrm{x}(\mathrm{I} / \mathrm{L}) \mathrm{xxxGL}$ motif, two structurally and functionally distinct proteins, $G$ protein and arrestin, can recognize similar activation signatures of $\mathrm{R}^{*}$ within the $\mathrm{E}(\mathrm{D}) \mathrm{RY}$ and $\operatorname{NPxxY}(\mathrm{x})_{5,6} \mathrm{~F}$ motifs. In the case of $\mathrm{G}$ protein, $\mathrm{G} \alpha \mathrm{CT}$ binds within the cytoplasmic crevice of $\mathrm{R}^{*}$, and this information is communicated down the long $\alpha 5$ helix of $\mathrm{G} \alpha$ to the nucleotidebinding site, where GDP/GTP exchange occurs ${ }^{1}$. Recent work has suggested that the flexibility of $\mathrm{G}_{\mathrm{t}} \alpha \mathrm{CT}$ allows it to rapidly enter the binding crevice, and after a process of mutual conformational adaptation, both $\mathrm{G}_{\mathrm{t}} \alpha \mathrm{CT}$ and the cytoplasmic crevice of the receptor gain a well-defined structure ${ }^{33}$. Our data indicate that arrestin partially mimics this process. The finger loop is highly flexible in basal arrestin $8,15,16,18,23$, and interaction with the binding crevice of $R^{\star}$ induces structuring in the form of a reverse turn. Comparison of crystal structures shows how the finger loop elongates in the progression of arrestin activation from basal to pre-activated to $\mathrm{R}^{*}$-bound (Fig. 3d-f). During arrestin activation, the phosphorylated receptor C-terminus forms an extended $\beta$-sheet with strand VI near the finger loop, which is held in place by hydrogen bonding to two phosphate moieties (Fig. 3e $)^{13}$. This initial interaction of arrestin with the phosphorylated receptor could stabilize and orient the flexible finger loop for tight binding to the crevice of $\mathrm{R}^{\star}$. Formation of the high-affinity complex pulls the finger loop further apart and may partially melt the $\beta$-sheet composed of strands $\mathrm{V}$ and VI. This transformation allows arrestin to block access of G protein to the receptor (signal shut-off) and may also induce other changes in the arrestin protein that promote scaffolding of signalling proteins (arrestin-mediated signalling).

In summary, the remarkable homology seen between $\mathrm{G} \alpha \mathrm{CT}$ and ArrFL suggests that the $(\mathrm{E} / \mathrm{D}) \mathrm{x}(\mathrm{I} / \mathrm{L}) \mathrm{xxxGL}$ motif evolved in both $G$ protein and arrestin to allow recognition and interaction with the same binding crevice presented by the active GPCR. In spite of these similarities, we also identify pronounced differences, namely a larger hydrophobic interaction surface in the case of $\mathrm{G}_{\mathrm{t}} \alpha \mathrm{CT}$, which may contribute to the distinct biological functions of $\mathrm{G}$ proteins and arrestins.

\section{Methods}

Peptide synthesis. All peptide experiments in this study were verified at least twice with peptide lots from two different manufacturers (Selleckchem and GenScript). Peptides from Selleckchem and GenScript were purchased with 95\% purity as verified by analytical high-performance liquid chromatography and Tandem Mass Spectrometry. Peptides of rod photoreceptor arrestin ( ${ }^{67}$ YGQEDIDVMGL ${ }^{77}$, ArrFL-1), $\beta$-arrestin-1/2 ( ${ }^{63 / 64}$ YGREDLDVLGL $^{73 / 74}$, ArrFL-2/3), wild-type $\mathrm{G}_{\mathrm{t}} \alpha\left({ }^{340} \mathrm{IKENLKDCGLF}^{350}, \mathrm{G}_{\mathrm{t}} \alpha \mathrm{CT}\right)$ and the high-affinity version of $\mathrm{G}_{\mathrm{t}} \alpha\left({ }^{340} \mathrm{ILENLKDCGLF}^{350}, \mathrm{G}_{\mathrm{t}} \alpha \mathrm{CT}-\mathrm{HA}\right)$ were synthesized without any modifications. The $\mathrm{G}_{\mathrm{s}} \alpha$ peptide $\left({ }^{384} \mathrm{QRMHLRQYELL}{ }^{394}, \mathrm{G}_{\mathrm{s}} \alpha \mathrm{CT}\right)$ was synthesized with or without N-terminal acetylation, which showed no difference in experiments. 

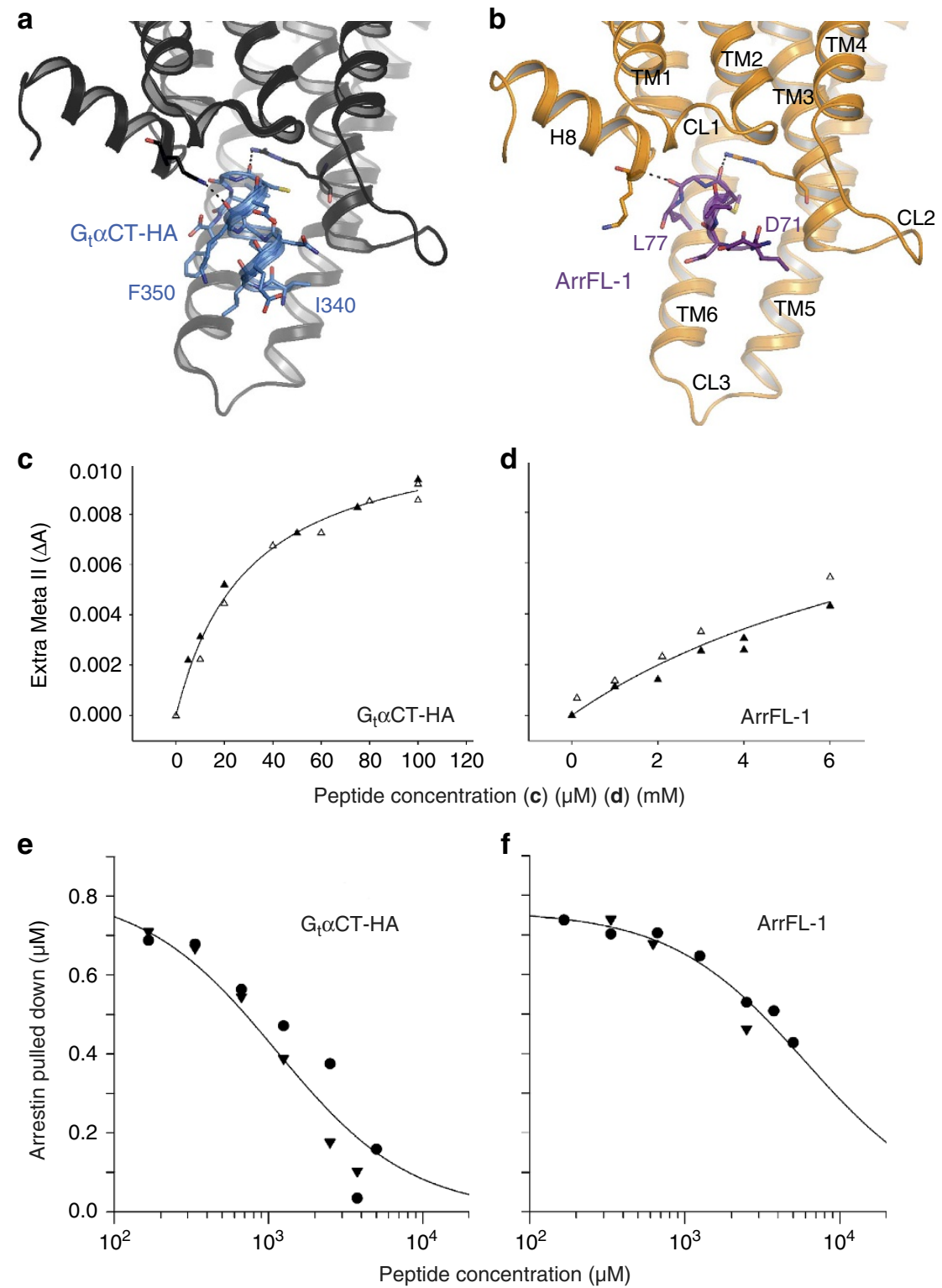

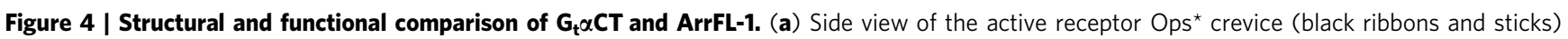

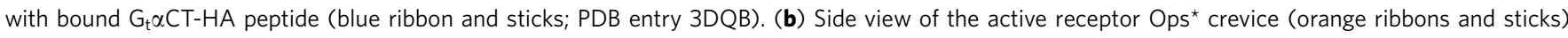
with bound ArrFL-1 peptide (purple ribbon and sticks). (c) Stabilization of Meta II by the high-affinity peptide $\mathrm{G}_{\mathrm{t}} \alpha \mathrm{CT}$-HA and (d) ArrFL-1 peptide. (e) Competition against rod photoreceptor arrestin for binding to phosphorylated opsin by $\mathrm{G}_{t} \alpha \mathrm{CT}-\mathrm{HA}$ and (f) ArrFL-1 peptide. In panels c-f, data points from independent experiments are represented as differently shaped symbols. The peptide titration experiments which measure Meta II-stabilization (c,d and Supplementary Fig. $9 a, b, c)$ yield an apparent $K_{D}$, from which the true $K_{D}$ value of peptide binding to Meta II can be derived. Further experimental details are given in the Methods.

Purification and crystallization of complexes. Rod outer segment (ROS) disc membranes containing rhodopsin were isolated from frozen bovine retina by a multi-step sucrose density gradient centrifugation procedure ${ }^{34}$. All procedures were carried out under dim red light, and retinas were sourced from a local slaughterhouse. Opsin was generated by exposing the isolated ROS membranes to a bright orange light in the presence of hydroxylamine. Membranes containing rhodopsin or opsin were stripped of peripheral proteins by washing three times with $4 \mathrm{M}$ urea ${ }^{21,35}$. Stripped membranes were subsequently solubilized in buffer A (20mM 1,3-Bis[tris(hydroxymethyl) methylamino]propane, $\mathrm{pH} 7.5,1.25 \%$ n-octylb-D-glucoside) at a concentration of about $200 \mu \mathrm{M}$ receptor. All peptides were solubilized in buffer A and the final $\mathrm{pH}$ was readjusted to 7.5 .

Synthetic peptides were added at a 12:1 molar ratio of peptide to protein (final receptor concentration $\sim 150 \mu \mathrm{M}$ ). Rhodopsin/peptide mixtures were kept on ice for $5 \mathrm{~min}$ and illuminated $(530 \mathrm{~nm})$ for $60 \mathrm{~s}$. The protein/peptide mixtures were crystallized in sitting drops by vapour diffusion at $277 \mathrm{~K}$ using 24 -well Limbro and 96-well MRC plates. The protein-to-reservoir-solution-ratio was optimized from 1:0.1 to 1:2 using a Mosquito crystallization robot (TTP Labtech). The reservoir solution contained $3.2-3.5 \mathrm{M}\left(\mathrm{NH}_{4}\right)_{2} \mathrm{SO}_{4}$ in $0.1 \mathrm{M}$ 2-(N-morpholino) ethanesulfonic acid or sodium acetate at $\mathrm{pH}$ 6.0. Crystals appeared within 7 days and grew for a further 5 days to reach dimensions $0.3 \times 0.3 \times 0.4 \mathrm{~mm}^{3}$. Crystals were then transferred under red light to cryoprotectant consisting of $10-15 \%$ trehalose in crystallization buffer and immediately frozen in liquid nitrogen. Ultraviolet-visible absorption spectra of the crystals were measured offline before $\mathrm{X}$-ray irradiation at $100 \mathrm{~K}$ using a HR2000 (OceanOptics) on a microspectrophotometer setup at beamline ID29S (ESRF) ${ }^{36}$ (Supplementary Fig. 2).

Data collection and structure analysis. Diffraction data collections of more than 500 crystals were performed at $100 \mathrm{~K}$ using synchrotron X-ray sources from ESRF and BESSY II. The best diffraction data for the highest resolution of Ops ${ }^{\star}-$ ArrFL-1 was collected at synchrotron beamline ID29 (ref. 37) at ESRF with a Pilatus 6M detector at $0.9791 \mathrm{~nm}$ wavelength. The crystal-to-detector distance was fixed at $521 \mathrm{~mm}$ for Ops ${ }^{*}$-ArrFL-1. All images were indexed, integrated and scaled using the XDS programme package ${ }^{38}$ and the CCP4 programme SCALA ${ }^{39,40}$. Ops ${ }^{*}-$ ArrFL-1 crystals belonged to the rhombohedral space group H32 (for Ops ${ }^{*}$-ArrFL1: $a=242.62 \AA, b=242.62 \AA, c=110.22 \AA, \alpha=90^{\circ}, \beta=90^{\circ}, \gamma=120^{\circ}$ ). Table 1 
summarizes the statistics for crystallographic data collection and structural refinement. Initial phases for Ops ${ }^{*}-\mathrm{ArrFL}-1$ were obtained by the conventional molecular replacement protocol (rotation, translation, rigid-body fitting) using the Ops $^{*}-\mathrm{G}_{\mathrm{t}} \alpha \mathrm{CT}$ structure (PDB accession $3 \mathrm{DQB}^{3}$ ) without $\mathrm{G}_{\mathrm{t}} \alpha \mathrm{CT}$ peptide as the initial search model, which was used in the programme Phaser ${ }^{41}$. A simulated annealing procedure with the resulting model was performed using a slow-cooling protocol and a maximum likelihood target function, energy minimization and B-factor refinement by the programme PHENIX ${ }^{42}$, which was carried out in the resolution range of 40.04-2.75 for the Ops ${ }^{*}$-ArrFL-1 structure. After the first round of refinements, the ArrFL-1 peptide and $\beta$-D-octylglucopyranoside in the ligand-binding pocket were clearly visible in the electron density of both $\sigma_{\mathrm{A}}$-weighted $2 F_{o}-F_{c}$ maps, as well as in the $\sigma_{\mathrm{A}}$-weighted simulated annealing omitted density maps for Ops ${ }^{\star}-$ ArrFL-1. Ops ${ }^{\star}-$ ArrFL-1 was modelled with TLS refinement ${ }^{43}$ (TLS - for Translation, Libration (small movements) and Screwrotation of a group of atoms) using anisotropic temperature factors for all atoms. Restrained, individual B-factors were refined, and the crystal structure was finalized by the CCP4 programme REFMAC5 (ref. 44) and other programmes of the CCP4 suite ${ }^{39}$. The final model has agreement factors $R_{\text {free }}$ and $R_{\text {cryst }}$ of 25.1 and $21.7 \%$, for $\mathrm{Ops}^{*}$-ArrFL-1. Manual rebuilding of the Ops*-ArrFL-1 model and electron density interpretation were performed after each refinement cycle using the programme $\mathrm{COOT}^{45}$. Structure validation was performed with the programmes PHENIX $^{42}$, SFCHECK $^{46}$, PROCHECK ${ }^{47}$, WHAT_CHECK ${ }^{48}$ and RAMPAGE ${ }^{49}$. Potential hydrogen bonds and van der Waals contacts were analysed using the programmes HBPLUS ${ }^{50}$ and LigPlot + (ref. 51). All crystal structure superpositions of backbone $\alpha$-carbon traces were performed using the CCP 4 programme $\mathrm{LSQKAB}^{39}$. All molecular graphics representations in this work were created using $\mathrm{PyMol}^{52}$.

Superscripts following receptor residue numbers denote $N$-terminus (NT), Helix 8 (H8), cytoplasmic loop and GPCR numbering according to Ballesteros and Weinstein ${ }^{53}$. The most conserved residue in a helix among rhodopsin-like GPCRs is designated $x .50$, where $x$ is the helix number. All other residues on the same helix are numbered relative to this reference residue.

Spectroscopic analysis of peptide binding. ROS membranes containing phosphorylated rhodopsin were prepared from frozen bovine retina (W.L. Lawson Company). Briefly, ROS membranes were isolated using a multi-step sucrose density centrifugation procedure. Phosphorylation of the rhodopsin by the associated native rhodopsin kinase was achieved by exposing the ROS to white light in the presence of adenosine triphosphate $(8 \mathrm{mM})$ and $\mathrm{MgCl}_{2}(2 \mathrm{mM})$. After $2 \mathrm{~h}$, hydroxylamine $(20 \mathrm{mM})$ was added to convert all rhodopsin photoproducts to opsin. The membranes containing phosphorylated opsin (OpsP) were then washed several times to remove the hydroxylamine and peripheral proteins and finally regenerated with 11-cis-retinal. These procedures have been recently described in detail $^{54}$. Peptides were solubilized in $100 \mathrm{mM}$ HEPES buffer, and the final $\mathrm{pH}$ was adjusted to 8.0. 'Extra Meta II' analysis was performed at $0^{\circ} \mathrm{C}$ using a Shimadzu UV3000 instrument ${ }^{30}$. Note that all peptides used for crystallization were first functionally verified by the absorbance 'Extra Meta II' assay. Samples consisted of $10 \mu \mathrm{M}$ ROS-P in $100 \mathrm{mM}$ HEPES buffer $\mathrm{pH} 8.0$ and increasing concentrations of peptide. The photo-flash activated $\sim 20 \%$ of the rhodopsin. The titration data were fit to the expression $\left(B_{\max }{ }^{*} x / K_{\mathrm{D}}+x\right)$, where the final amplitude of the plots was fixed to the theoretical maximum absorbance change (that is, $100 \%$ Meta II stabilization). The resulting apparent $K_{\mathrm{D}}$ value was corrected for Meta I/Meta II equilibrium at $\mathrm{pH} 8.0$ (refs 30,55 ). The true $K_{\mathrm{D}}$ value of peptide binding to Meta II was derived from the apparent $K_{\mathrm{D}}$ value, as described by Schleicher et al..$^{30,55}$, using a Meta II/Meta I ratio of $\sim 0.1$ (refs 30,55).

Centrifugal pull-down binding assay. The preparation of phosphorylated opsin from ROS membranes is described in the method section 'Spectroscopic analysis of peptide binding'. The bovine rod photoreceptor arrestin mutant C63A/C128S/ C143A/W194F/A366C was expressed in E. coli and purified using two-step ion exchange chromatography (heparin and Q sepharose, GE Helathcare Life Sciences $^{56,57}$. This single-cysteine mutant $(50 \mu \mathrm{M})$ was labelled with a tenfold molar excess of $N, N^{\prime}$-dimethyl- $N$-(Iodoacetyl)- $N^{\prime}$ - (7-Nitrobenz-2-Oxa-1,3-Diazol-4yl)Ethylenediamine (NBD, Life Technologies) for $3 \mathrm{~h}$ at room temperature. Free label was removed by buffer exchange using centrifugal concentrators followed by size exclusion chromatography (Sephadex G-15, $500 \mu \mathrm{l}$ void volume). NBDlabelled arrestin A366C (A366NBD) is functionally equivalent to wild type ${ }^{58}$. Peptides were solubilized in $50 \mathrm{mM}$ HEPES buffer, and the $\mathrm{pH}$ was adjusted to 7.0. Peptide competition against arrestin A366NBD $(1 \mu \mathrm{M})$ for binding to OpsP $(2 \mu \mathrm{M})$ was measured by a centrifugal pull-down assay ( $50 \mathrm{mM}$ HEPES, pH 7.0,

$\left.22^{\circ} \mathrm{C}\right)^{54,58}$. Briefly, arrestin and OpsP were mixed with increasing concentrations of peptide (120 $\mathrm{ll}$ sample volume). Following an equilibration period of $\sim 30 \mathrm{~min}$, samples were centrifuged at $16,000 \mathrm{~g}$ for $10 \mathrm{~min}$ (room temperature). The absorbance spectrum of each supernatant was then measured. Given the redshifted absorbance of the NBD label $\left(\lambda_{\max }=500 \mathrm{~nm}\right)$ and its defined extinction coefficient $\left(0.025 \mu \mathrm{M}^{-1} \mathrm{~cm}^{-1}\right)$, the concentration of arrestin remaining in the supernatant could be accurately determined. The $K_{\mathrm{D}}$ of peptide binding to OpsP was determined by fitting the peptide titration data to the expression: $y=s^{*}\left(1-\left(P /\left(P+K_{\mathrm{Dp}}{ }^{*}\left(1+A / K_{\mathrm{Da}}\right)\right)\right)\right.$, where $P$ is the peptide concentration, $K_{D p}$ is the $K_{\mathrm{D}}$ of the peptide, $A$ is the arrestin concentration $(1 \mu \mathrm{M}), K_{\mathrm{Da}}$ is the $K_{\mathrm{D}}$ of arrestin binding to OpsP $(400 \mathrm{nM})^{54}$. The scaling factor $s$ was necessary as $y$ axis values were not normalized.

FTIR spectroscopy. FTIR difference spectroscopy is a sensitive monitor of the structural influence of peptide binding on the active receptor ${ }^{32,33}$. Briefly, FTIR difference spectra of rhodopsin $(\sim 1 \mathrm{mM})$ were recorded at room temperature and $\mathrm{pH} 5.8$ to ensure exclusive formation of the active receptor conformation upon illumination $\left(\lambda_{\max }=532 \mathrm{~nm}\right)$. A separate measurement was performed under identical conditions but in the presence of an excess of peptide $\left(5 \mathrm{mM}\right.$ for $\mathrm{G}_{\mathrm{t}} \alpha \mathrm{CT}$, $14 \mathrm{mM}$ for all other peptides). The peptide binding spectra (difference of the difference spectra recorded in the presence and absence of peptide) were calculated after normalization of all spectra to the difference bands between 1,500 and $1,300 \mathrm{~cm}^{-1}$. Similar FTIR measurements were carried out for the R135L receptor mutant and the ArrFL-1 peptide. Mutant rhodopsin was expressed in COS-1 cells using the eukariotic expression vector pMT4 (refs 59,60). After regeneration with $30 \mu \mathrm{M} 11$-cis-retinal the protein was purified in $1 \% n$-D-octyl-b-D-glucopyranoside using the 1D4 antibody bound to sepharose resin. Rhodopsin was eluted from the resin with rhodopsin-derived C-terminal peptide 340-348. The protein was thereafter incorporated into egg-PC lipid vesicles at a ratio of 1:100 (rhodopsin/ lipid) by dialysis against $20 \mathrm{mM}$ bis-tris propane, $130 \mathrm{mM} \mathrm{NaCl}, 1 \mathrm{mM} \mathrm{MgCl}_{2}$, pH 7.0 (ref. 61).

\section{References}

1. Hofmann, K. P. et al. A G protein-coupled receptor at work: the rhodopsin model. Trends Biochem. Sci. 34, 540-552 (2009).

2. Kisselev, O. G. et al. Light-activated rhodopsin induces structural binding motif in G protein alpha subunit. Proc. Natl Acad. Sci. USA 95, 4270-4275 (1998).

3. Scheerer, P. et al. Crystal structure of opsin in its G-protein-interacting conformation. Nature 455, 497-502 (2008).

4. Choe, H. W. et al. Crystal structure of metarhodopsin II. Nature 471, 651-655 (2011).

5. Rasmussen, S. G. et al. Crystal structure of the beta2 adrenergic receptor-Gs protein complex. Nature 477, 549-555 (2011).

6. Deupi, X. et al. Stabilized G protein binding site in the structure of constitutively active metarhodopsin-II. Proc. Natl Acad. Sci. USA 109, 119-124 (2012).

7. Arshavsky, V. Y. \& Burns, M. E. Photoreceptor signaling: supporting vision across a wide range of light intensities. J. Biol. Chem. 287, 1620-1626 (2012).

8. Hirsch, J. A., Schubert, C., Gurevich, V. V. \& Sigler, P. B. The 2.8 A crystal structure of visual arrestin: a model for arrestin's regulation. Cell 97, 257-269 (1999).

9. Han, M., Gurevich, V. V., Vishnivetskiy, S. A., Sigler, P. B. \& Schubert, C. Crystal structure of beta-arrestin at 1.9 A: possible mechanism of receptor binding and membrane Translocation. Structure 9, 869-880 (2001).

10. Sutton, R. B. et al. Crystal structure of cone arrestin at 2.3A: evolution of receptor specificity. J. Mol. Biol. 354, 1069-1080 (2005).

11. Zhan, X., Gimenez, L. E., Gurevich, V. V. \& Spiller, B. W. Crystal structure of arrestin-3 reveals the basis of the difference in receptor binding between two non-visual subtypes. J. Mol. Biol. 406, 467-478 (2011).

12. Kim, Y. J. et al. Crystal structure of pre-activated arrestin p44. Nature 497, 142-146 (2013).

13. Shukla, A. K. et al. Structure of active beta-arrestin-1 bound to a G-protein-coupled receptor phosphopeptide. Nature 497, 137-141 (2013)

14. Hanson, S. M. et al. Differential interaction of spin-labeled arrestin with inactive and active phosphorhodopsin. Proc. Natl Acad. Sci. USA 103, 4900-4905 (2006).

15. Sommer, M. E., Farrens, D. L., McDowell, J. H., Weber, L. A. \& Smith, W. C. Dynamics of arrestin-rhodopsin interactions: loop movement is involved in arrestin activation and receptor binding. J. Biol. Chem. 282, 25560-25568 (2007).

16. Kim, M. et al. Conformation of receptor-bound visual arrestin. Proc. Natl Acad. Sci. USA 109, 18407-18412 (2012).

17. Ostermaier, M. K., Peterhans, C., Jaussi, R., Deupi, X. \& Standfuss, J. Functional map of arrestin-1 at single amino acid resolution. Proc. Natl Acad. Sci. USA 111, 1825-1830 (2014).

18. Zhuang, T. et al. Involvement of distinct arrestin-1 elements in binding to different functional forms of rhodopsin. Proc. Natl Acad. Sci. USA 110, 942-947 (2012).

19. Sinha, A., Jones Brunette, A. M., Fay, J. F., Schafer, C. T. \& Farrens, D. L. Rhodopsin TM6 can interact with two separate and distinct sites on arrestin: evidence for structural plasticity and multiple docking modes in arrestinrhodopsin binding. Biochemistry 53, 3294-3307 (2014).

20. Park, J. H. et al. Opsin, a structural model for olfactory receptors? Angewandte Chemie. 52, 11021-11024 (2013). 
21. Park, J. H., Scheerer, P., Hofmann, K. P., Choe, H. W. \& Ernst, O. P. Crystal structure of the ligand-free G-protein-coupled receptor opsin. Nature 454, 183-187 (2008).

22. Standfuss, J. et al. The structural basis of agonist-induced activation in constitutively active rhodopsin. Nature 471, 656-660 (2011).

23. Feuerstein, S. E. et al. Helix formation in arrestin accompanies recognition of photoactivated rhodopsin. Biochemistry 48, 10733-10742 (2009).

24. Raman, D., Osawa, S. \& Weiss, E. R. Binding of arrestin to cytoplasmic loop mutants of bovine rhodopsin. Biochemistry 38, 5117-5123 (1999).

25. Marion, S., Oakley, R. H., Kim, K. M., Caron, M. G. \& Barak, L. S. A betaarrestin binding determinant common to the second intracellular loops of rhodopsin family G protein-coupled receptors. J. Biol. Chem. 281, 2932-2938 (2006).

26. Lan, H., Teeter, M. M., Gurevich, V. V. \& Neve, K. A. An intracellular loop 2 amino acid residue determines differential binding of arrestin to the dopamine D2 and D3 receptors. Mol. Pharmacol. 75, 19-26 (2009).

27. Rahmeh, R. et al. Structural insights into biased G protein-coupled receptor signaling revealed by fluorescence spectroscopy. Proc. Natl Acad. Sci. USA 109, 6733-6738 (2012).

28. Liu, J. J., Horst, R., Katritch, V., Stevens, R. C. \& Wuthrich, K. Biased signaling pathways in beta2-adrenergic receptor characterized by 19F-NMR. Science 335, 1106-1110 (2012)

29. Wacker, D. et al. Structural features for functional selectivity at serotonin receptors. Science 340, 615-619 (2013).

30. Schleicher, A., Kühn, H. \& Hofmann, K. P. Kinetics, binding constant, and activation energy of the $48-\mathrm{kDa}$ protein-rhodopsin complex by extrametarhodopsin II. Biochemistry 28, 1770-1775 (1989).

31. Hamm, H. E. et al. Site of G protein binding to rhodopsin mapped with synthetic peptides from the alpha subunit. Science 241, 832-835 (1988).

32. Vogel, R., Martell, S., Mahalingam, M., Engelhard, M. \& Siebert, F. Interaction of a $\mathrm{G}$ protein-coupled receptor with a $\mathrm{G}$ protein-derived peptide induces structural changes in both peptide and receptor: a Fourier-transform infrared study using isotopically labeled peptides. J. Mol. Biol. 366, 1580-1588 (2007).

33. Elgeti, M. et al. Precision vs flexibility in GPCR signaling. J. Am. Chem. Soc. 135, 12305-12312 (2013).

34. Papermaster, D. S. Preparation of retinal rod outer segments. Methods Enzymol. 81, 48-52 (1982).

35. Sachs, K., Maretzki, D. \& Hofmann, K. P. Assays for activation of opsin by all-trans-retinal. Methods Enzymol. 315, 238-251 (2000).

36. Royant, A. et al. Advances in spectroscopic methods for biological crystals. 1. Fluorescence lifetime measurements. J. Appl. Crystallogr. 40, 1105-1112 (2007).

37. de Sanctis, D. et al. ID29: a high-intensity highly automated ESRF beamline for macromolecular crystallography experiments exploiting anomalous scattering. J Synchrotron Radiat. 19, 455-461 (2012).

38. Kabsch, W. Xds. Acta Crystallogr. D Biol Crystallogr. 66, 125-132 (2010).

39. Collaborative Computational Project, N. The CCP4 suite: programs for protein crystallography. Acta Crystallogr. D Biol Crystallogr. 50, 760-763 (1994).

40. Evans, P. Scaling and assessment of data quality. Acta Crystallogr. D Biol. Crystallogr. 62, 72-82 (2006).

41. McCoy, A. J. et al. Phaser crystallographic software. J. Appl. Crystallogr. 40, 658-674 (2007).

42. Adams, P. D. et al. PHENIX: a comprehensive Python-based system for macromolecular structure solution. Acta Crystallogr. D Biol .Crystallogr. 66, 213-221 (2010).

43. Winn, M. D., Isupov, M. N. \& Murshudov, G. N. Use of TLS parameters to model anisotropic displacements in macromolecular refinement. Acta Crystallogr. D Biol. Crystallogr. 57, 122-133 (2001).

44. Vagin, A. A. et al. REFMAC5 dictionary: organization of prior chemical knowledge and guidelines for its use. Acta Crystallogr. D Biol. Crystallogr. 60, 2184-2195 (2004).

45. Emsley, P., Lohkamp, B., Scott, W. G. \& Cowtan, K. Features and development of Coot. Acta Crystallogr. D Biol. Crystallogr. 66, 486-501 (2010).

46. Vaguine, A. A., Richelle, J. \& Wodak, S. J. SFCHECK: a unified set of procedures for evaluating the quality of macromolecular structure-factor data and their agreement with the atomic model. Acta Crystallogr. D Biol. Crystallogr. 55, 191-205 (1999).

47. Laskowski, R. A., Moss, D. S. \& Thornton, J. M. Procheck: a program to check the stereo chemical quality of protein structures. J. Appl. Cryst. 26, 283-291 (1993).

48. Hooft, R. W., Vriend, G., Sander, C. \& Abola, E. E. Errors in protein structures. Nature 381, 272 (1996).

49. Lovell, S. C. et al. Structure validation by C alpha geometry: phi,psi and C beta deviation. Proteins 50, 437-450 (2003).

50. McDonald, I. K. \& Thornton, J. M. Satisfying hydrogen bonding potential in proteins. J. Mol. Biol. 238, 777-793 (1994).

51. Laskowski, R. A. \& Swindells, M. B. LigPlot + : multiple ligand-protein interaction diagrams for drug discovery. J. Chem. Informat. Model 51, 2778-2786 (2011).
52. DeLano, W. L. The PyMOL Molecular Graphics System (DeLano Scientific, 2002).

53. Ballesteros, J. A. \& Weinstein, H. Integrated methods for the construction of threedimensional models and computational probing of structure-function relations in G-protein coupled receptors. Methods Neurosci. 25, 366-428 (1995)

54. Sommer, M. E., Hofmann, K. P. \& Heck, M. Distinct loops in arrestin differentially regulate ligand binding within the GPCR opsin. Nat. Commun. 3, 995 (2012).

55. Parkes, J. H. \& Liebman, P. A. Temperature and $\mathrm{pH}$ dependence of the metarhodopsin I-metarhodopsin II kinetics and equilibria in bovine rod disk membrane suspensions. Biochemistry 23, 5054-5061 (1984).

56. Sommer, M. E., Smith, W. C. \& Farrens, D. L. Dynamics of arrestin-rhodopsin interactions: acidic phospholipids enable binding of arrestin to purified rhodopsin in detergent. J. Biol. Chem. 281, 9407-9417 (2006).

57. Gurevich, V. V. \& Benovic, J. L. Arrestin: mutagenesis, expression, purification, and functional characterization. Methods Enzymol. 315, $422-437$ (2000).

58. Sommer, M. E., Hofmann, K. P. \& Heck, M. Arrestin-rhodopsin binding stoichiometry in isolated rod outer segment membranes depends on the percentage of activated receptors. J. Biol. Chem. 286, 7359-7369 (2011).

59. Ferretti, L., Karnik, S. S., Khorana, H. G., Nassal, M. \& Oprian, D. D. Total synthesis of a gene for bovine rhodopsin. Proc. Natl Acad. Sci. USA 83, 599-603 (1986).

60. Sakmar, T. P. Rhodopsin: a prototypical G protein-coupled receptor. Progr. Nucleic Acid Res. Mol. Biol. 59, 1-34 (1998).

61. Elgeti, M., Kazmin, R., Heck, M., Morizumi, T. \& Ritter, E. et al. Conserved Tyr223(5.58) plays different roles in the activation and G-protein interaction of rhodopsin. J. Am. Chem. Soc. 133, 7159-7165 (2011).

\section{Acknowledgements}

We thank Anja Koch and Brian Bauer for assistance in purifying reagents and opsin purification. We are grateful to Uwe Müller, Manfred Weiss and the scientific staff of the BESSY-MX/Helmholtz Zentrum Berlin für Materialien und Energie at beamlines BL14.1, BL14.2 and BL14.3 operated by the Joint Berlin MX-Laboratory at the BESSY II electron storage ring (Berlin-Adlershof, Germany) and the scientific staff of the European Synchrotron Radiation Facility (ESRF, Grenoble) at beamlines ID14-1, ID23-1, ID23-2, ID14-4 and ID29, where the data were collected, for continuous support. We thank Antoine Royant for extensive support with the usage of the microspectrophotometer on the crystals at the ID29S-Cryobench (ESRF, Grenoble). This work was supported by grants from the Deutsche Forschungsgemeinschaft (SFB740 to K.P.H., M.H., and P.W.H.; SO1037/1-1,2 to M.E.S.; WO1908/2-1, Hi 1502/1-1 to P.W.H.; SFB1078-B6 to P.S.), European Research Council (Advanced Investigator Grant (ERC-2009/249910TUDOR to K.P.H.), European Synchrotron Radiation Facility (ESRF to P.S.), DFG Cluster of Excellence 'Unifying Concepts in Catalysis' (Research Field D3/E3-1 to P.S.).

\section{Author contributions}

M.S. and P.S. conceived, planned and designed the project; P.S. coordinated the project; M.S. performed opsin preparation, functional analysis and crystallization; D.v.S., M.S. and P.S. performed ultraviolet-visible absorption spectroscopy on crystals; M.S. and P.S. performed X-ray diffraction data collection; P.S. performed structural analysis; M.S., A.R., P.W.H. and P.S. performed bioinformatics analyses; F.B. and M.H. performed the 'Extra Meta II' assays; M.E.S. performed peptide competition experiments; M.E., R.K. and F.J.B. performed and analysed FTIR experiments; M.S., A.R., P.W.H. and P.S. prepared all figures; all authors contributed to data analysis and interpretation; M.S., K.P.H., P.W.H., M.E.S. and P.S. wrote the paper.

\section{Additional information}

Accession codes: The atomic coordinates and structure factors have been deposited in the Protein Data Bank under accession code 4PXF.

Supplementary Information accompanies this paper at http://www.nature.com/ naturecommunications

Competing financial interests: The authors declare no competing financial interests.

Reprints and permission information is available online at http://npg.nature.com/ reprintsandpermissions/

How to cite this article: Szczepek, M. et al. Crystal structure of a common GPCR-binding interface for G protein and arrestin. Nat. Commun. 5:4801 doi: 10.1038/ncomms5801 (2014).

This work is licensed under a Creative Commons AttributionNonCommercial-ShareAlike 4.0 International License. The images or other third party material in this article are included in the article's Creative Commons license, unless indicated otherwise in the credit line; if the material is not included under the Creative Commons license, users will need to obtain permission from the license holder to reproduce the material. To view a copy of this license, visit http:// creativecommons.org/licenses/by-nc-sa/4.0/ 